Supporting information for

Auger Dynamics in InP/ZnSe/ZnS Quantum Dots Having Pure and Doped Shells

Anh T. Nguyen, ${ }^{1}$ Paul Cavanaugh, ${ }^{1}$ Ilan Jen-La Plante, ${ }^{2}$ Christian Ippen, ${ }^{2}$ Ruiqing $\mathrm{Ma}^{2}$ and David F. Kelley $^{1 *}$

${ }^{1}$ Chemistry and Biochemistry, University of California Merced, 5200 North Lake Road, Merced, CA 95343

${ }^{2}$ Nanosys Inc., 233 S. Hillview Dr, Milpitas, CA 95035

Trion decay kinetics.
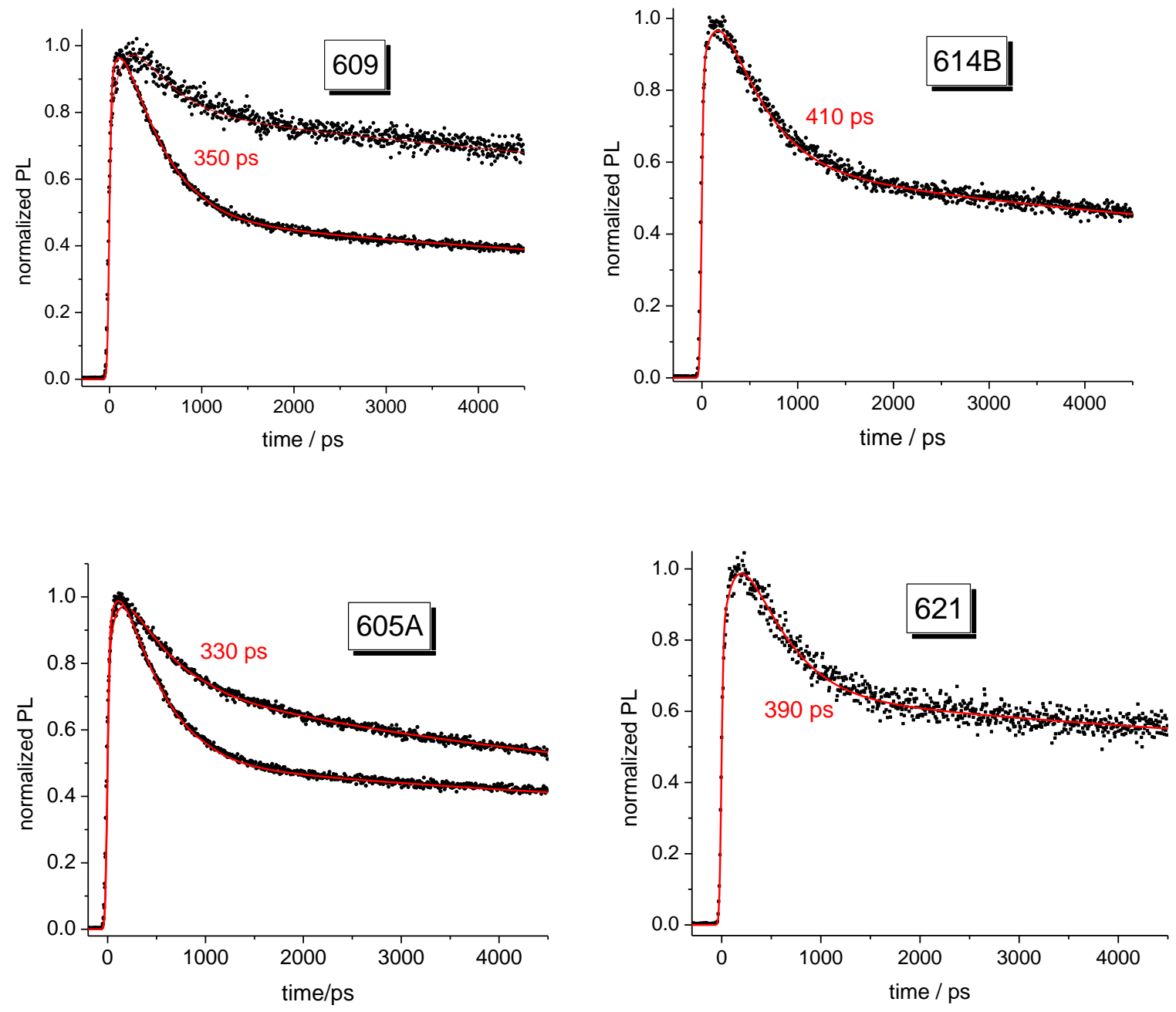

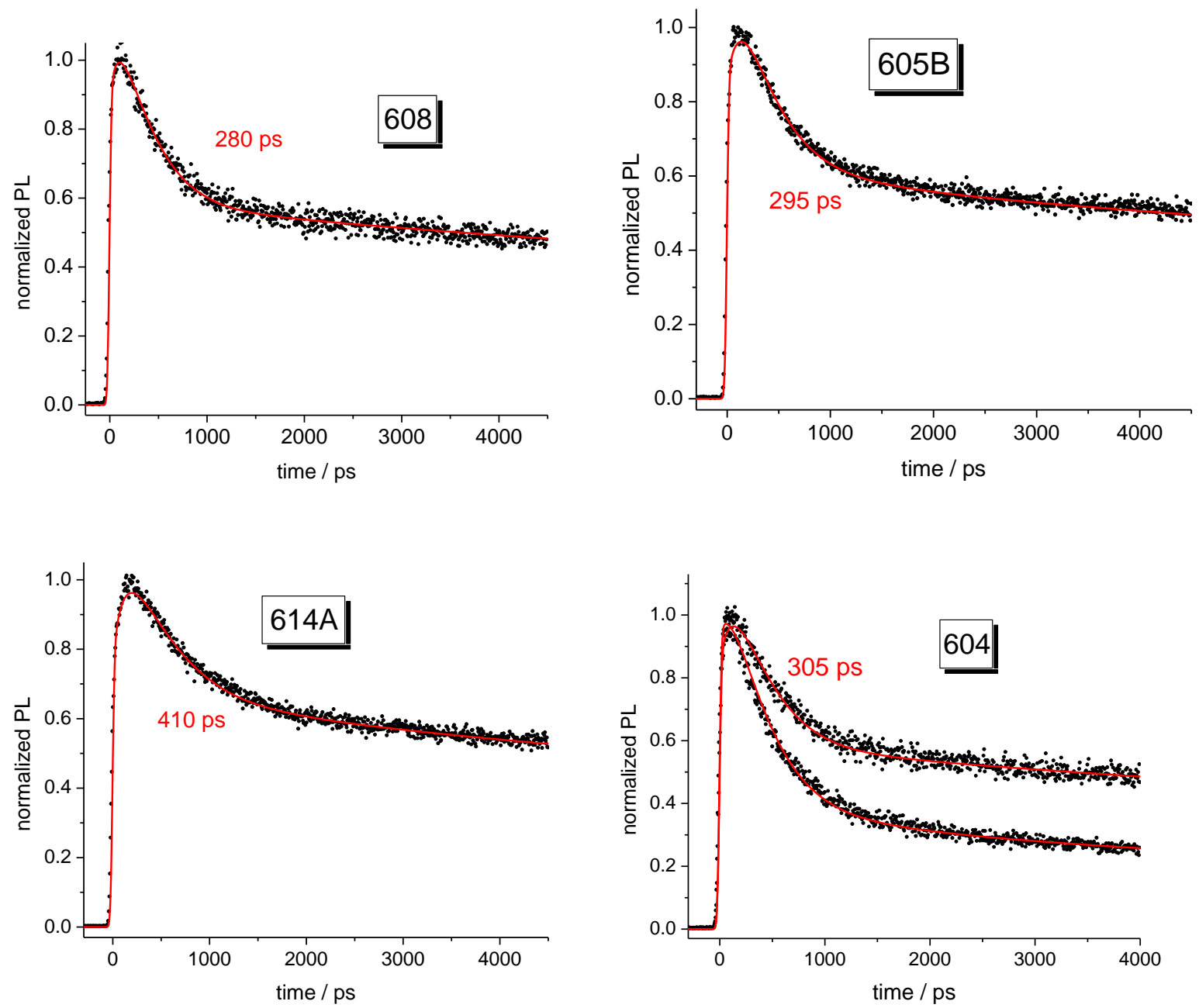

Figure S1. Negative trion PL decays following lithium triethylborohydride photoreduction, for the different QDs, as indicated. The negative trion decay times are also indicated. In the cases of the 605A, 609 and 604 QDs, trion decays were measured following photoreduction with differing amounts of borohydride and fits to the same trion decay times are shown.

\section{Biexciton kinetics.}

With the present experimental conditions, the lowest exciton has an absorbance of about 0.2 and following $387 \mathrm{~nm}$ excitation, $\Delta \mathrm{A}$ values are about 0.05 . Making the crude approximation that the absorption follows Poisson statistics (uniform intensity throughout the sample) we get $0.15 / 0.20=\mathrm{P}(0)+$ $1 / 2 \mathrm{P}(1)$, where $\mathrm{P}(\mathrm{n})=\lambda^{\mathrm{n}} / \mathrm{n}$ ! $\mathrm{e}^{-\lambda}$. This gives $\lambda=0.52$ and $59 \%$ absorb zero, $31 \%$ absorb 1 and $8 \%$ absorb 3 photons. For a concentration of $0.3 \mu \mathrm{M}$, there are $1.8 \times 10^{14} \mathrm{QDs} / \mathrm{cm}^{3}$. With a pump power of $0.4 \mathrm{~mW}$ (a $1 \mathrm{kHz}$ rep rate and chopping every other pulse gives an incident power of about $0.2 \mathrm{~mW}$ after the chopper) and a spot size of $1 \mathrm{~mm}\left(\right.$ area $\left.=8 \times 10^{-3} \mathrm{~cm}^{2}\right)$ this gives $10^{14}$ photons $/ \mathrm{pulse} / \mathrm{cm}^{2}$. At $387 \mathrm{~nm}, \sigma=$ $3 \times 10^{-14} \mathrm{~cm}^{2}$, and the flux is $10^{14}$ photons $/ \mathrm{cm}^{2}$, so about 3 photons per QD at the front face and progressively less through the sample. The TA bleach decays obtained under these conditions are shown below. 

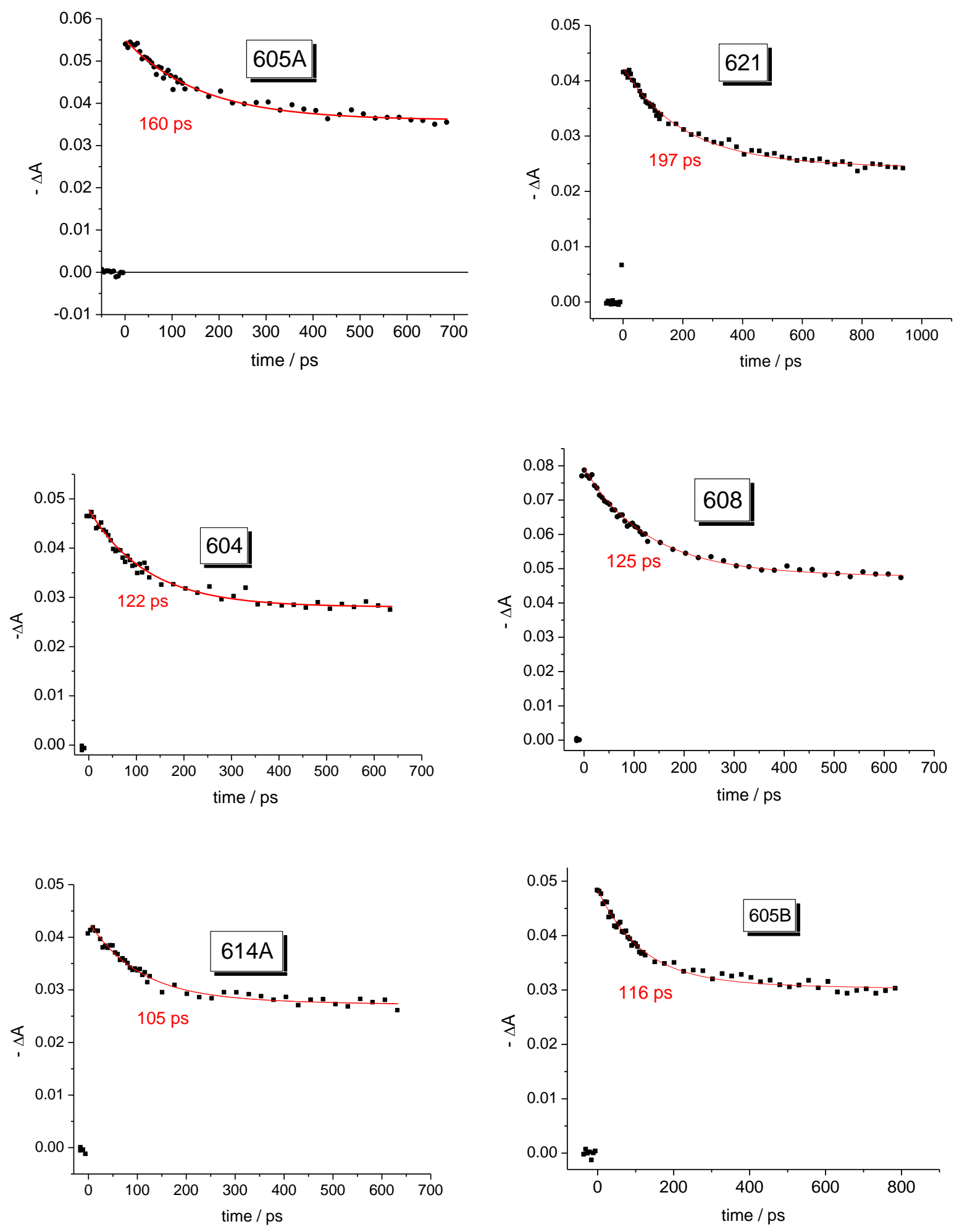

Figure S2. Transient absorption bleach recovery kinetics for the nonstoichiometric QDs, as indicated. The fitting to a single exponential biexciton decay is also shown and the time constant indicated. 


\section{Radiative dynamics in exciton, trions and biexcitons.}

We first establish the relative absorption and PL oscillator strengths involving transitions of the ground state $(\mathrm{QD})$, exciton $(\mathrm{X})$, biexciton $(\mathrm{XX})$, charged $\mathrm{QD}\left(\mathrm{QD}^{-}\right)$and negative trion $\left(\mathrm{T}^{-}\right)$. These relative oscillator strengths are taken primarily from a series of papers by Efros and coworkers. These papers are the classic papers on the "dark exciton" 1,2 the biexciton spectroscopy paper, ${ }^{3}$ and the trion spectroscopy paper. ${ }^{4}$

The oscillator strengths (or transition probabilities) are given in terms of $\mathrm{KP}^{2}$, where $\mathrm{K}$ is the overlap integral and $\mathrm{P}$ is the Kane (momentum) matrix element; $\mathrm{P}$ is proportional to the matrix element of the dipole moment operator ${ }^{5}$ and $\mathrm{P}^{2}$ is therefore proportional to the oscillator strength. If we assume particle-in-asphere wavefunctions, all dependencies on $\mathrm{K}$ can be ignored and we will do this in the simple treatment below. With this approximation, all the transition probabilities can be expressed in units $\mathrm{KP}^{2}$, which is the same for all the cases that we consider.

We can measure the integrated extinction coefficient for the QD $\rightarrow \mathrm{X}$ transition, so we start by considering both the QD $\rightarrow \mathrm{X}$ and $\mathrm{X} \rightarrow$ QD transitions. The basic electronic structure of a II-VI or II-V exciton is shown figure $\mathrm{S} 3$.

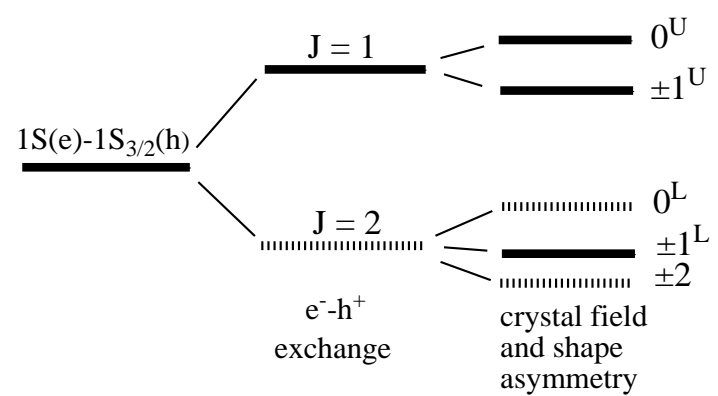

Figure S3. Angular momentum fine structure energy levels of the lowest exciton in CdSe QDs. Optically allowed (forbidden) levels are indicated with solid (dotted) lines.

In all of the lowest exciton states, the electron is in the s-orbitals of the conduction band and the hole is in p-orbitals of the valence band. In the simplest case of spherical zincblende particles, all the $J=2$ states (the $\pm 2, \pm 1^{\mathrm{L}}$ and $0^{\mathrm{L}}$ ) are degenerate and dark and the $\mathrm{J}=1$ states (the $0^{\mathrm{U}}$ and $\pm 1^{\mathrm{U}}$ ) have allowed transitions from the ground state. The $J=2$ and $J=1$ states correspond to pure triplets and singlets, respectively. This is easily seen in for the \pm 2 states in terms of an electron-in-orbitals picture. The upper arrow represents a conduction band (indium s orbitals) electron and the lower represents a valence band (phosphorous $\mathrm{p}$ orbitals) hole in the $\mathrm{p}_{1}$ orbital.

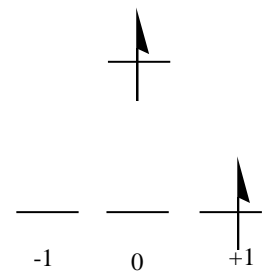

The hole is in the $m_{1}=1$ orbital and the spins are aligned, so $m_{s}=1$, for a total $j_{z}=2$. This is a pure triplet, so radiation to the ground state is spin forbidden. Other states are constructed from linear combinations of different hole spins (either spin up or spin down) in the different p orbitals. The coefficients of these $11 \mathrm{~m}_{1} \mathrm{~s} \mathrm{~m}_{\mathrm{s}}>$ states that comprise the angular momentum fine structure states are the Clebsch-Gordon coefficients. (Reference 1 expands these states in terms of the Wigner 3-j symbols.) The splitting between the $\mathrm{J}=1$ and $\mathrm{J}=2$ states is simply two times the electron-hole exchange integral. Any loss of spherical symmetry splits the $\mathrm{J}$ states into different sublevels and mixes the bright $\pm 1^{\mathrm{U}}$ and dark $\pm 1^{\mathrm{L}}$ states, giving 
finite oscillator strength to the latter. In the $\mathrm{InP} / \mathrm{ZnSe}$, this perturbation is relatively small and for approximately spherical particles, the $\pm 1^{\mathrm{L}}$ has only a small fraction of the total transition oscillator strength. ${ }^{6}$ The energy spacings of these states and the finite but low oscillator strength in the $\pm 1^{\mathrm{L}}$ state is fundamentally why InP/ZnSe QDs have very long radiative lifetimes. The energy splitting between the dark exciton $\left(\mathrm{J}_{z}=\right.$ \pm 2 ) and the lowest allowed state $\left(J_{z}= \pm 1^{L}\right)$ is small, about $5-10 \mathrm{meV} .^{6-8}$ The fully allowed $\mathrm{J}=1$ states are split from the $\mathrm{J}=2$ states by exchange interaction and is therefore much higher in energy, about $100 \mathrm{meV} .{ }^{6}$ Photoexcitation is primarily to the higher states and PL comes primarily from the bright states that are thermally accessible, the $\pm 1^{\mathrm{L}}$ and $\pm 1^{\mathrm{U}}$. Most of the room temperature thermal population is in the dark \pm 2 and $0^{\mathrm{L}}$ states, with some is in the partially allowed $\pm 1^{\mathrm{L}}$ states. The molecular spectroscopy way of stating this is that the population is mostly in the triplets, so radiation to the ground state is spin forbidden and therefore slow. This can be made more quantitative through the Einstein relations. ${ }^{5,9}$

Exciton extinction coefficients.

To use the Einstein relations, we must know the integrated extinction coefficient of the lowest exciton. The approach taken here uses the known QD volumes and optical properties of the bulk materials at a very high energies to determine the QD photon cross section or extinction coefficient at that high energy (350 $\mathrm{nm})$. This treatment of the extinction coefficients makes several assumptions and approximations.

We ignore the presence of the $\mathrm{ZnS}$ shell in the treatment of InP/ZnSe/ZnS QDs. The absorption spectra are evaluated at $350 \mathrm{~nm}, 450 \mathrm{~nm}$ and at the lowest exciton. The bandgap of $\mathrm{ZnS}$ is sufficiently large that absorption into the $\mathrm{ZnS}$ shell should not contribute at these wavelengths. Furthermore, the band offsets are such that there is little electron or hole density in the $\mathrm{ZnS}$ part of the shell. So ignoring the $\mathrm{ZnS}$ shell is a good approximation. We evaluate the QD absorption at $350 \mathrm{~nm}$ and equate this to the absorption of equal volumes of the bulk materials. This assumes that there are no quantum confinement effects at this wavelength. This is more than $1 \mathrm{eV}$ above the exciton, so this is a good approximation.

The starting points are the particle core and shell volumes and the wavelength-dependent "absorption coefficient", $\alpha$, of the bulk materials. At $350 \mathrm{~nm}, \alpha=2.0 \mathrm{e}+5 \mathrm{~cm}^{-1}$ for ZnSe and $\alpha=6.8 \mathrm{e}+5 \mathrm{~cm}^{-1}$ for InP. ${ }^{10}$, 11 (The value is much higher for InP because $350 \mathrm{~nm}$ is much further above the bandgap than $\mathrm{ZnSe}$, and therefore InP has a much larger density of states at that energy.) The QD photon cross section is then given by $\sigma / \mathrm{cm}^{2}=\left(\text { Volume } / \mathrm{cm}^{3}\right)^{*}\left(\alpha / \mathrm{cm}^{-1}\right)$. The volumes depend critically on the core and shell dimensions and the calculations below use values in table 1 and reference 12. The green particles in reference 12 have a $2.0 \mathrm{~nm}$ core and a $1.85 \mathrm{~nm}$ thick $\mathrm{ZnSe}$ shell. Thus, the InP and $\mathrm{ZnSe}$ volumes are $4.2 \mathrm{e}-21$ and $9.3 \mathrm{e}-20$ $\mathrm{cm}^{3}$, respectively. Thus, considering both the InP and ZnSe components for the green QDs we get $\sigma_{\text {grn }}(350$ $\mathrm{nm})=2.14 \mathrm{e}-14 \mathrm{~cm}^{-1}$. The molar extinction coefficient is related to the photon cross section by $\sigma / \mathrm{cm}^{2}=$ $\left(2303 / \mathrm{N}_{\mathrm{Av}}\right) \varepsilon / \mathrm{M}^{-1} \mathrm{~cm}^{-1}$. Thus, the corresponding $350 \mathrm{~nm}$ extinction coefficient is $5.58 \mathrm{e}+6 \mathrm{M}^{-1} \mathrm{~cm}^{-1}$. An analogous calculation for the red QDs takes the core diameter to be $3.0 \mathrm{~nm}$ and the ZnSe shell thickness to be $2.5 \mathrm{~nm}$. We get $\sigma_{\mathrm{red}}(350 \mathrm{~nm})=6.05 \mathrm{e}-14 \mathrm{~cm}^{-1}$, corresponding to an extinction coefficient of $1.58 \mathrm{e}+7$ $\mathrm{M}^{-1} \mathrm{~cm}^{-1}$. From the above $350 \mathrm{~nm}$ values and the absorption spectra (i.e., the ratios of absorbances at different wavelengths), photon cross sections or extinction coefficients at other wavelengths can be calculated. For the green QDs, we get $\varepsilon_{350}=5.58 \mathrm{e}+6, \varepsilon_{450}=\varepsilon_{508}=4.79 \mathrm{e}+5$. For the red QDs, we get $\varepsilon_{350}$ $=1.58 \mathrm{e}+7, \varepsilon_{450}=1.95 \mathrm{e}+6, \varepsilon_{604}=6.44 \mathrm{e}+5$.

Radiative lifetimes.

For nondegenerate states, the radiative rate is given by ${ }^{9}$

$A=\frac{8 \pi 0.2303 n_{f}^{3}}{N_{a} c^{2} n_{a}}\left\langle v_{f}^{-3}\right\rangle^{-1} \int \frac{\varepsilon(v)}{v} d v \approx 2.88 \times 10^{-9} n^{2}\left\langle\tilde{v}_{f}^{2}\right\rangle \int \varepsilon(\tilde{v}) d \tilde{v}$

equation 1.

which may be approximately written as 


$$
A=\frac{8 \pi 0.2303 n_{f}^{3}}{N_{a} n_{a}} \frac{c}{\lambda^{3}} \int \frac{\varepsilon(\lambda)}{\lambda} d \lambda \approx 2.88 \times 10^{-15} \frac{n^{2}}{\lambda^{4}} \int \varepsilon(\lambda) d \lambda \approx 2.88 \times 10^{-15} \frac{n^{2}}{\lambda^{4}} \varepsilon\left(\lambda_{\max }\right) \Delta \lambda \quad \text { equation } 2 .
$$

where $\Delta \lambda$ is the full width half maximum of the fluorescence peak, typically about $35 \mathrm{~nm}$. The approximation going from equation 1 to 2 is correct to within about $6 \%$ for a Gaussian band shape. The important point is that the radiative rate is proportional to the integral of the exciton peak extinction coefficient. To evaluate equation 2 , we take $\mathrm{n}^{2}=2, \lambda=630 \mathrm{~nm}=630 \mathrm{e}-9 \mathrm{~m}, \varepsilon\left(\lambda_{\max }\right)=6.4 \mathrm{e}+5$ and $\Delta \lambda=35$ $\mathrm{nm}=35 \mathrm{e}-9 \mathrm{~m}$. This gives $\mathrm{A}=8.05 \mathrm{e}+8 \mathrm{~s}^{-1}$, or a radiative lifetime of $1.23 \mathrm{~ns}$ for a two-level, nondegenerate system having this integrated extinction coefficient. We note that this treatment ignores local field factors. This is because the local field factors enter into calculations of the absorption and emission oscillators in the same way, and therefore cancel. The observed radiative lifetime for the standard red QDs is about 25 times longer. In the 1996 Efros paper, ${ }^{1}$ this corresponds to a total oscillator strength of $4 / 3 \mathrm{KP}^{2}$, into the combination of the \pm 1 and $0^{\mathrm{U}}$ states. The main reason for this very long observed lifetime is that most of the population is in non-emissive states associated with different terms in the angular momentum fine structure. The dark and bright state populations are the dominant factor in controlling the observed lifetime. However, the important inverse proportionality that comes out of this treatment is that in an InP/ZnSe QD having these dimensions, a nondegenerate oscillator strength of $4 / 3 K P^{2}$ corresponds to a radiative lifetime of $1.2 \mathrm{~ns}$. This will be of use in evaluating the biexciton and trion radiative lifetimes.

Negative trions.

The negatively charged QD has an electron in the conduction band and therefore is a ${ }^{2} \mathrm{~S}_{1 / 2}$ state. The negative trion has the conduction band electrons are paired and a hole in one of the p-orbitals. Thus, the trion has orbital angular momentum of either 0 or 1 and therefore has ${ }^{2} \mathrm{P}_{1 / 2}$ and ${ }^{2} \mathrm{P}_{3 / 2}$ states, as depicted below. The lowest energy trion state is the $\mathrm{J}=3 / 2$ state, with the $\mathrm{J}=1 / 2$ state at slightly higher energy. Thus, the absorption $\left(\mathrm{QD}^{-} \rightarrow \mathrm{T}^{-}\right)$and $\mathrm{PL}\left(\mathrm{T}^{-} \rightarrow \mathrm{QD}^{-}\right)$oscillators are nondegenerate, making this a simple problem. Radiative recombination of the $\mathrm{J}=3 / 2$ trion leaves an unpaired conduction band electron, a $\mathrm{J}=$ $1 / 2$ state. This is a $\Delta \mathrm{J}=1$ transition and is fully allowed; there is no dark exciton for the trion. ${ }^{4}$ In the language of molecular spectroscopy, the transitions between ground and excited states are spin-allowed doublet-doublet transitions.

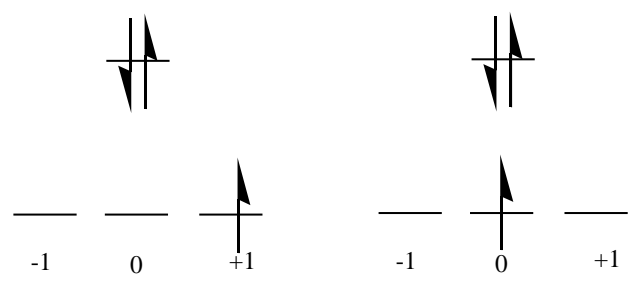

The negatively charged particle absorption oscillator strength (integrated extinction coefficient) is affected by the presence of the conduction band electron because of conduction band state filling, lowering the extinction coefficient by a factor of two. This can also be seen by comparison of the oscillator strength expressions in the Efros papers. ${ }^{1,3,4}$ Equation 28 of the 1996 Efros paper gives a total transition probability (to all of the fine structure levels) of $4 / 3 \mathrm{KP}^{2}$ for the unchaged ground state to exciton transition. In comparison, the negative trion oscillator strengths come from integration of equations 64 and 65 of reference 4 , which gives the absorption and PL oscillator strengths of $2 / 3 \mathrm{KP}^{2}$. This is also explicitly stated in equation 66 of reference 4 . Thus, ignoring difference in the overlap integrals, the negative trion radiative lifetime is expected to be what a factor of two longer than what is calculated from equation 2 , about $1.20 \mathrm{x}$ $2.0=2.4$ ns. This also follows directly from equation 2, above. Trion PL corresponds to a fully allowed, 
nondegenerate transition having half the extinction coefficient as the neutral QD. It therefore has twice the radiative lifetime as one calculates from the neutral QD extinction coefficient.

\section{Biexcitons.}

The Efros biexciton paper ${ }^{3}$ summarizes the absorption $(\mathrm{X} \rightarrow \mathrm{XX})$ and PL $(\mathrm{XX} \rightarrow \mathrm{X})$ transiton oscillator strengths in figures 6 and 5, respectively of reference 3. Figure 6 gives the exciton to biexciton transition probability of $2 / 3 \mathrm{KP}^{2}$. This is just the same as the $\mathrm{QD}^{-} \rightarrow \mathrm{T}^{-}$transition and a factor of two less than the $\mathrm{QD} \rightarrow \mathrm{X}$ transition. The reason is the same for the trion and the biexciton: conduction band state filling. Both the negatively charged QD and the exciton have an electron in the conduction band - they are half filled. So the intensity of transitions putting another electron there is down by a factor of two. For the XX radiative lifetime, if all the population is taken to be in the lowest energy biexciton $0^{-}$state, the PL is dominated by the transition to the exciton $\pm 1^{\mathrm{L}}$ states, see figure 5 of reference 3 . Each transition has an oscillator strength of $2 / 3 \mathrm{KP}^{2}$, so together the transitions give a total oscillator strength of $4 / 3 \mathrm{KP}^{2}$. It is important to note that this is a factor of two more oscillator strength and hence a factor of two shorter than the radiative lifetime than the negative trion. The biexciton radiative lifetime is expected to be about 1.2 ns.

\section{Statistical scaling.}

It is sometimes (incorrectly) stated that the radiative rates in InP-based or CdSe-based QDs exhibit "statistical scaling", i.e., that the radiative rate scales proportional to the product of the number of electrons and holes. The negative trion has two electrons, so statistical scaling predicts that the radiative rate of the negative trion would be twice that of the neutral exciton, and this is sometimes stated in journal papers. The above considerations show that this idea is incorrect; it completely ignores electron spin in general and specifically ignores the slow radiative rate of the lowest exciton fine structure state, the pure-triplet dark exciton. This point is particularly obvious when one compares the case of the singlet exciton with that of the negative trion. The singlet exciton has a lone conduction band electron that can radiatively recombine with the hole. The negative trion has two conduction band electrons that are paired with opposite spins. However, only one of the paired conduction band electrons has the correct spin to radiatively recombine with the hole. So, by any analysis, the statistical scaling model is inapplicable to II-VI and III-V QDs.

We note that the statistical scaling model is a reasonable approximation for radiative recombination in $\mathrm{PbS}$ and $\mathrm{PbSe}$ nanocrystals. ${ }^{13}$ The reason why it approximately works for the lead chalcogenides has to do with their crystal structure and electronic states. $\mathrm{PbS}$ and $\mathrm{PbSe}$ have a rocksalt crystal structure and in that lattice, the electronic structure has four degenerate, minimum bandgap positions (referred to as "valleys"), at the four $\mathrm{L}$ points of the band structure diagram. The valleys are weakly coupled and radiative recombination occurs only when both the electron and hole are in the same valley. The probability of this happening is proportional to the number of electrons and holes. Despite the fact that this is clearly stated in the original literature, this model is occasionally misapplied to zincblende semiconductor QDs.

\section{References.}

1. Efros, A. L.; Rosen, M.; Kuno, M.; Nirmal, M.; Norris, D. J.; Bawendi, M., Band-Edge Exciton in Quantum Dots of Semiconductors With a Degenerate Valence Band: Dark and Bright Exciton States. Phys. Rev. B 1996, 54, 4843-4856.

2. Sercel, P. C.; Efros, A. L., Band-Edge Exciton in CdSe and Other II-VI and III-V Compound Semiconductor Nanocrystals - Revisited. Nano Lett. 2018, 18, 4061-4068.

3. Rodina, A. V.; Efros, A. L., Band-edge Biexciton in Nanocrystals of Semiconductors with a Degenerate Valence Band. Phys. Rev. B 2010, 82, 125324-125337. 
4. Shabaev, A.; Rodina, A. V.; Efros, A. L., Fine Structure of the Band-Edge Excitons and Trions in CdSe/CdS Core/Shell Nanocrystals. Phys. Rev. B 2012, 86, 205311-205325.

5. Kelley, A. M., Condensed-Phase Molecular Spectroscopy and Photophysics. Wiley: Hoboken, NJ, 2013.

6. Biadala, L.; Siebers, B.; Beyazit, Y.; Tessier, M. D.; Dupont, D.; Hens, Z.; Yakovlev, D. R.; Bayer, M., Band-Edge Exciton Fine Structure and Recombination Dynamics in InP/ZnS Colloidal Nanocrystals. ACS Nano 2016, 10, 3356-3364.

7. Brodu, A.; Chandrasekaran, V.; Scarpelli, L.; Buhot, J.; Masia, F.; Ballottin, M. V.; Severijnen, M.; Tessier, M. D.; Dupont, D.; Rabouw, F. T., et al., Fine Structure of Nearly Isotropic Bright Excitons in InP/ZnSe Colloidal Quantum Dots. J. Phys. Chem. Lett. 2019, 10, 5468-5475.

8. $\quad$ Brodu, A.; Ballottin, M. V.; Buhot, J.; van Harten, E. J.; Dupont, D.; La Porta, A.; Prins, P. T.; Tessier, M. D.; Versteegh, M. A. M.; Zwiller, V., et al., Exciton Fine Structure and Lattice Dynamics in InP/ZnSe Core/Shell Quantum Dots. ACS Photonics 2018, 5, 3353-3362.

9. Strickler, S. J.; Berg, R. A., Relationship between Absorption Intensity and Fluorescence Lifetime of Molecules. J. Chem. Phys. 1962, 37, 814-822.

10. Adachi, S.; Taguchi, T., Optical properties of ZnSe. Phys. Rev. B 1991, 43, 9569 - 9577.

11. Adachi, S., Optical dispersion relations for GaP, GaAs, GaSb, InP, InAs, InSb, AlxGa1-xAs, and In1-xGaxAsyP1-y. J. App. Phys. 1989, 66, 6030-6040.

12. Nguyen, A. T.; Jen-La Plante, I.; Ippen, C.; Ma, R.; Kelley, D. F., Extremely Slow Trap-Mediated Hole Relaxation in Room-Temperature InP/ZnSe/ZnS Quantum Dots. J. Phys. Chem. C 2021, 125, 41104118

13. McGuire, J. A.; Joo, J.; Pietryga, J. M.; Schaller, R. D.; Klimov, V. I., New Aspects of Carrier Multiplication in Semiconductor Nanocrystals. Acc. Chem. Res 2008, 41, 1810. 